Cite this: J. Mater. Chem. B, 2014, 2 , 3315

Received 11th February 2014 Accepted 20th March 2014

DOI: $10.1039 / c 4 t b 00226 a$

www.rsc.org/MaterialsB

\section{Chitosan scaffolds with a shape memory effect induced by hydration}

\author{
Cristina O. Correia ${ }^{a b}$ and João F. Mano*ab
}

\begin{abstract}
We demonstrate that chitosan-based porous scaffolds can present a shape memory effect triggered by hydration. The shape memory effect of non-crosslinked (CHTO) and genipin-crosslinked (CHT1) scaffolds was followed by innovative hydromechanical compressive tests and dynamic mechanical analysis (DMA), while the sample was immersed in varying compositions of water-ethanol mixtures. By dehydration with higher contents of ethanol, the vitreous-like nature of the amorphous component of chitosan allows the fixation of the temporary shape of the scaffold. The presence of water disrupts intermolecular hydrogen bonds permitting large-scale segmental mobility of the chitosan chains upon the occurrence of glass transition and thus the recovery of the permanent shape of a pre-deformed scaffold. Results showed that chitosan possesses shape memory properties, characterized by a fixity ratio above 97.2\% for $\mathrm{CHTO}$ and above $99.2 \%$ for $\mathrm{CHT1}$ and a recovery ratio above $70.5 \%$ for $\mathrm{CHTO}$ and $98.5 \%$ for CHT1. In vitro drug delivery studies were also performed to demonstrate that such devices can also be loaded with molecules. We show that the developed chitosan scaffolds are candidates of biomaterials for applications in minimally invasive surgery for tissue regeneration or for drug delivery.
\end{abstract}

\section{Introduction}

The rapid progress in the development of surgical techniques, especially in minimally invasive surgery, leads to more complex requirements for modern implants. Besides important properties like biocompatibility and, ideally in many cases, degradability, the shape memory effect (SME) as a novel functionality of polymers might enable the development of novel types of medical devices..$^{1-3}$ Shape memory polymers (SMPs) offer novel materials-based devices for solving scientific challenges due to their demonstrated ability to actively undergo geometric transformations upon exposure to environmental stimuli.,5

A shape-memory polymer can be deformed by application of an external stress and fixed in a second shape, the temporary shape. This temporary shape is retained until the shaped body is exposed to an appropriate stimulus, which induces the recovery of the original shape. ${ }^{4}$ The existence of physical or chemical crosslinking points is required for the SME to recover the initial shape after deformation and fixation. ${ }^{6}$ SMPs can utilize glass transition temperature $\left(T_{\mathrm{g}}\right)^{7}$ and/or melting points $\left(T_{\mathrm{m}}\right)^{8}$ as the deformation/fixing temperatures. SMP contain a network architecture consisting of netpoints that are connected

${ }^{a} 3 B$ 's Research Group - Biomaterials, Biodegradables and Biomimetics, Department of Polymer Engineering, University of Minho, Headquarters of the European Institute of Excellence on Tissue Engineering and Regenerative Medicine; AvePark, Zona Industrial da Gandra S. Cláudio do Barco, 4806-909 Caldas das Taipas, Guimarães, Portugal

${ }^{b} I C V S / 3 B$ 's, PT Government Associate Laboratory, Braga/Guimarães, Portugal. E-mail: jmano@dep.uminho.pt with stimuli-sensitive macromolecular chains. The netpoints determine the permanent shape and the segment chains are used as a kind of molecular switch. Above the transition temperature the switchable segments give flexibility for the deformation; under this temperature the segments give stiffness for shape fixation..$^{9}$ Besides the temperature, shape memory devices can also use other stimuli, such as hydration, ${ }^{10}$ light, ${ }^{11}$ electromagnetic $^{12}$ or electrical. ${ }^{13}$ To date, the most attractive SMPs are triggered by temperature. ${ }^{14}$ Thermal-responsive SMPs are normally driven by external heat, which is an issue for applications with restricted temperature ranges like in the biomedical field. ${ }^{15}$ Nonthermally induced shape memory polymers eliminate the temperature constrains and enable the manipulation of the shape recovered under ambient temperature. ${ }^{16}$

Recent studies have shown that environmental conditions such as humidity can substantially influence conformational mobility of macromolecular chains, and thus the shape memory properties of some polymers. ${ }^{17-20}$ These findings motivated the development of the concept of a moisture triggered SME.

Water or solvent-driven shape recovery effects have been observed in SMPs having glass transitions as switching transition. This type of polymer absorbs water, and this affects their mechanical and physical properties. ${ }^{21,22}$ By disrupting intramolecular hydrogen bonds and acting as a plasticizer, water reduces the glass transition temperature and hence effectively allows for room temperature actuation. ${ }^{23}$

Shape memory polymers are ideal candidates for biomedical applications in which a temporary shape has to be preserved 
until the device is placed in the cavity to be filled, allowing minimally invasive surgical procedures. ${ }^{24,25}$ However most of the polymers studied with shape memory properties are nonbiodegradable. It is often important that medical polymers present a biodegradable behavior in vivo, that would avoid a secondary surgery to remove them from the body. Polymers with degradability and shape memory capability are multifunctional materials. Recently, controlled drug release has been added to the list of functionalities of SMPs. ${ }^{\mathbf{1 , 2 6}}$

Chitosan (CHT) is a partially N-deacetylated derivative of chitin. ${ }^{27}$ Considerable attention has been given to this polymer due to its advantages like low cost, large-scale availability, antimicrobial activity, non-toxicity, biodegradability, and biocompatibility. ${ }^{27-29}$ Chitosan can be processed into scaffolds using different processing techniques to be used in tissue engineering. ${ }^{30,31}$

The goal of this work is to develop CHT-based scaffolds with shape memory properties. Previously it was found that CHT can undergo a glass transition by the action of hydration..$^{32-34}$ By combining such an effect with the maintenance of the structural integrity of the system (provided by crosslinking or due to the intrinsic semi-crystalline structure) we hypothesize that we could develop new three-dimensional devices in which the recovery of the geometry can be induced by hydration. To control this parameter the tests were conducted in waterethanol mixtures with distinct compositions. Moreover, the capability of drug delivery was studied in order to achieve multifunctional devices that combine the SME, biodegradability and drug delivery ability.

\section{Materials and methods}

\subsection{Materials}

Chitosan (CHT) of medium molecular weight $\left(M_{\mathrm{w}}=190000-\right.$ $310000,75-85 \%$ degree of deacetylation, viscosity $200-800 \mathrm{cps}$ ) was purchased from Sigma Aldrich. Before being used CHT was purified using a reprecipitation method. CHT powder was dissolved at a concentration of $1 \%(\mathrm{w} / \mathrm{v})$ in $2 \%(\mathrm{v} / \mathrm{v})$ aqueous acetic acid and precipitated with a $\mathrm{NaOH}$ solution (final $\mathrm{pH} \sim 8$ ). The CHT flakes were washed with distilled water until neutralization and dehydrated with ethanol. Finally, the CHT flakes were frozen and lyophilized. Genipin was a product of Wako Chemicals. Congo Red and gelatin was purchased from Sigma Aldrich. All other chemicals were of reagent grade and were used as received.

\subsection{Methods}

2.2.1 Chitosan scaffold preparation. CHT was dissolved in an aqueous acetic acid solution $2 \%(\mathrm{v} / \mathrm{v})$ to a concentration of $3 \%(\mathrm{w} / \mathrm{v})$ under stirring until homogeneity was reached. For the crosslinked system (CHT1), 3 wt $\%$ genipin (chitosan-genipin = $100 / 3 \mathrm{w} / \mathrm{w}$ ) was added to the CHT solution under stirring. Noncrosslinked CHT scaffolds (CHT0) were prepared directly from the original CHT solutions, with no addition of genipin. In order to obtain scaffolds with a cylindrical shape, chitosan solutions were cast into silicone tubes. For the case of CHT1, the tubes with the genipin solution were maintained under stirring for $6 \mathrm{~h}$ at $37^{\circ} \mathrm{C}$. Then, all solutions were frozen for 1 day at $-80{ }^{\circ} \mathrm{C}$. After freeze-drying, the scaffolds were neutralized with a solution of $\mathrm{NaOH}(1 \mathrm{M})$ and then freeze-dried again. The tubes were then cut into small pieces to obtain a scaffold dimension of $7 \mathrm{~mm} \times \varnothing 5 \mathrm{~mm}$.

2.2.2 Scanning electron microscopy (SEM). A NanoSEM FEI Nova 200 (FEG/SEM) scanning electron microscope was used to study the surface and the morphology of the samples. Prior to observation all samples were coated by gold sputtering for 2 minutes at a current of $15 \mathrm{~mA}$.

2.2.3 Pore size and porosity measurements. The pore size of the samples was observed using SEM, by which we could derive the corresponding mean value and standard deviation $(N$ $=15$ ). The porosity of the scaffolds was obtained from the density of chitosan (assumed to be $\rho=1.342 \mathrm{~g} \mathrm{~cm}^{-3}$ ) and the density of the scaffold using

$$
\text { porosity }(\%)=\frac{V_{\mathrm{m}}-\left(W_{\mathrm{m}} / \rho\right)}{V_{\mathrm{m}}} \times 100
$$

where, $V_{\mathrm{m}}$ is the total volume of the chitosan scaffolds $\left(\mathrm{cm}^{3}\right)$ and $W_{\mathrm{m}}$ is the mass of the scaffold (g).

2.2.4 Swelling of chitosan scaffolds in water-ethanol mixtures. The swelling of CHT scaffolds in mixtures of waterethanol was determined by immersing previously weighed CHT scaffolds in mixtures of these solvents at compositions varying from pure water to pure ethanol at room temperature for $4 \mathrm{~h}$. It was confirmed that after a $4 \mathrm{~h}$ period the scaffolds had reached their swelling equilibrium. After $4 \mathrm{~h}$, the swollen samples were blotted with a filter paper to remove the adsorbed solvent and weighed immediately. The swelling ratio $(S)$ was calculated using the following equation:

$$
S(\mathrm{wt} \%)=\frac{\left(w-w_{0}\right)}{w_{0}} \times 100
$$

where, $w$ is the weight of the swollen sample and $w_{0}$ is the weight of the dry sample. Each swelling experiment was performed in triplicate.

2.2.5 X-ray diffraction measurements. The crystallinity of non-crosslinked chitosan scaffolds was investigated by X-ray diffraction (XRD) analysis performed with a Bruker D8 Discover model. XRD patterns were examined in the region of $5-65^{\circ}$ with a step size of $0.02^{\circ}$ for $2 \theta$ and a counting time of $2 \mathrm{~s}$ per step.

2.2.6 Dynamic Mechanical Analysis (DMA). Dynamic mechanical analysis was performed using Tritec 2000B equipment (Triton Technology, UK). The measurements were carried out at room temperature $\left(20^{\circ} \mathrm{C}\right)$. Chitosan scaffolds were tested at constant frequency $(1 \mathrm{~Hz})$ following the changes in the storage modulus $\left(E^{\prime}\right)$ and loss factor $(\tan \delta)$ as a function of the water content. The dried samples of known geometry were placed in a Teflon ${ }^{\circledR}$ reservoir and immersed in a defined volume of ethanol $\left(V_{\text {eth }}=270 \mathrm{ml}\right)$ and kept under the testing constant strain amplitude $(30 \mu \mathrm{m})$ for $30 \mathrm{~min}$. After this step, $E^{\prime}$ reached an equilibrium value. Finally, water was pumped into the reservoir at a constant flow rate $\left(Q=14 \mathrm{ml} \mathrm{min}^{-1}\right)$, providing a time $(t)$ dependent change in the content of water described by the following equation: 


$$
\text { water }(\operatorname{vol} \%)=\frac{Q t}{Q t+V_{\text {eth }}}
$$

In this measurement $E^{\prime}$ should be taken as an apparent value as during introduction of water the geometry of the sample continuously changes due to swelling and the calculation of this parameter used the initial geometry of the sample.

2.2.7 Hydromechanical cyclic compressive tests. The compressive tests of the developed scaffolds were performed using a Universal tensile testing machine (Instron 4505 Universal Machine, USA). The tests were performed under compressive loading, by performing uniaxial compression, using a crosshead speed of $2 \mathrm{~mm} \mathrm{~min}^{-1}$ at room temperature. The results presented are the average of at least three specimens. Each compressive test was performed with the scaffolds hydrated in water-ethanol mixtures with distinct compositions. Young's modulus $(E)$ was determined from the initial slope of the stress-strain curves.

For hydromechanical cyclic compressive tests, the samples were first hydrated and deformed up to different maximum strains $\left(\varepsilon_{\mathrm{m}}=10,20,30\right.$ and $\left.60 \%\right)$. After the test and maintaining the strain, the stress was then held constant while the sample is dehydrated by immersing in ethanol for $20 \mathrm{~min}$, whereby the temporary shape is fixed. Then the stress was completely removed to obtain the sample in its temporary shape, even after complete evaporation of ethanol. Finally, the samples were immersed in different mixtures of water-ethanol, varying from pure water to pure ethanol and the shape recovery was monitored.

In the hydromechanical cyclic compressive test, the shape memory capability of the SMP is typically characterized by the shape fixity ratio $\left(R_{\mathrm{f}}\right)$ and the shape recovery ratio $\left(R_{\mathrm{r}}\right) \cdot R_{\mathrm{f}}$ characterizes the ability of a system to fix its temporary shape and $R_{\mathrm{r}}$ is the recoverability of the permanent shape. $R_{\mathrm{f}}$ and $R_{\mathrm{r}}$ values can be calculated according to the following equations:

$$
\begin{gathered}
R_{\mathrm{f}}(\%)=\frac{\varepsilon_{\mathrm{u}}}{\varepsilon_{\mathrm{m}}} \times 100 \\
R_{\mathrm{r}}(\%)=1-\frac{\varepsilon_{\mathrm{p}}}{\varepsilon_{\mathrm{m}}} \times 100
\end{gathered}
$$

where $\varepsilon_{\mathrm{m}}$ is the applied maximum strain, $\varepsilon_{\mathrm{u}}$ is the fixed strain after unloading and $\varepsilon_{\mathrm{p}}$ is the permanent strain after induced recovery. The measurements were performed with a digital micrometer (in triplicate).

The scaffold recovery along time was evaluated to see how the hydrated scaffolds recovered after being submitted to compression loads. The scaffolds were initially hydrated and then compressed up to different maximum strains $\left(\varepsilon_{\mathrm{m}}=10,20\right.$ and $30 \%)$. Then, the samples were dehydrated to fix the temporary shape. The samples were immersed again in water and their height was measured at different time points.

2.2.8 In vitro drug delivery studies. Congo Red (CR) was used as a model molecule to investigate the loading and release ability of the scaffold. CR is water soluble and presents low solubility in ethanol. ${ }^{35}$
In drug delivery studies it is important to know the incorporation efficiency (IE) of the scaffolds. The incorporation efficiency can be measured by the ratio of the drug available for release and the initial loaded drug,

$$
\mathrm{IE}=\frac{\text { Drug available for release }}{\text { Loaded drug }} \times 100
$$

The scaffold was immersed in a solution of CR in PBS of $1 \mathrm{mg}$ $\mathrm{ml}^{-1}$ for $1 \mathrm{~h}$. The loaded drug was estimated using the variation of the mass of the scaffold during swelling. Then, the loaded scaffolds were compressed $\left(\varepsilon_{\mathrm{m}}=30 \%\right)$ and dehydrated followed by immersion in $5 \mathrm{ml}$ of PBS under sonication at $37^{\circ} \mathrm{C}$ for 5 days to induce a forced release, obtaining the maximum drug release. During this process we did not observe any substantial degradation of the scaffold. Such an experiment permitted the estimation of the total drug available for release in the scaffolds.

For the loading procedure, the samples were immersed in a solution of CR in PBS of $5 \mathrm{mg} \mathrm{ml}^{-1}$ for a period of $1 \mathrm{~h}$. After complete hydration the samples were compressed $\left(\varepsilon_{\mathrm{m}}=30 \%\right)$ and dehydrated for $20 \mathrm{~min}$ in ethanol, followed by the evaporation of the solvent. After the deformation and fixation of the temporary shape, both CHT0 and CHT1 were immersed in $5 \mathrm{ml}$ of PBS ( $\mathrm{pH}$ 7.4). The vials containing the scaffolds were kept under agitation (at approximately $60 \mathrm{rpm}$ ) in a water bath at $37^{\circ} \mathrm{C}$. At pre-established periods of time, aliquots of $1 \mathrm{ml}$ of the supernatant were taken out and replaced with an equal volume of fresh PBS to maintain the volume constant during the release study. The amount of Congo Red was quantified spectrophotometrically, measuring the UV absorbance at $498 \mathrm{~nm}$ (Synergie HT, Bio-Tek, USA) and using a calibration curve generated by absorption measurements of CR solutions with pre-defined concentrations. For exemplification of the release profile the scaffolds were immersed in a gelatin block to release CR. We prepared a $1 \%$ gelatin solution and heated for $30 \mathrm{~min}$ at $50{ }^{\circ} \mathrm{C}$. Then, the solution was stored at $4-8{ }^{\circ} \mathrm{C}$ in the refrigerator until use.

\section{Results and discussion}

\subsection{Morphological characterization}

Adequate scaffolds used for tissue engineering should typically exhibit a homogenous microstructure, suitable pore size distribution and high porosity. Such a microstructure should act as an extracellular matrix analogue, functioning as a necessary template for host infiltration and as a physical support to guide the differentiation and proliferation of cells into the targeted functional tissue or organ. ${ }^{36}$

The SEM images in Fig. 1 show the morphological characteristics of the non-crosslinked (CHT0) and crosslinked (CHT1) scaffolds at the microscale level. The scaffold revealed an interconnected porous structure, with pore sizes in the range of 200-350 $\mu \mathrm{m}$ for CHT0. CHT1 shows a more regular morphology with smaller pores in the range of $100-250 \mu \mathrm{m}$. The pore walls in CHT1 are thinner and the amount of pores is higher; thus the overall surface area for possible cell attachment is larger for CHT1. The CHT0 and CHT1 scaffolds have a density of 

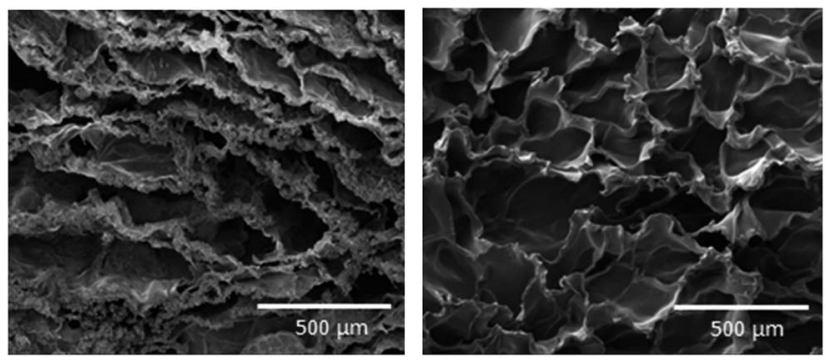

Fig. 1 SEM images of (left) porous non-crosslinked (CHTO) and (right) crosslinked (CHT1) chitosan scaffolds.

$0.155 \mathrm{~g} \mathrm{~cm}^{-3}$ and $0.076 \mathrm{~g} \mathrm{~cm}^{-3}$, respectively. The decrease in the density of CHT1 results in a porosity of $94 \%$ which is higher than the $88 \%$ obtained for CHT0.

\subsection{Swelling of chitosan scaffolds in water-ethanol mixtures}

In the presence of suitable solvents, chemically or physically crosslinked polymer networks do not dissolve, but absorb limited amounts of solvent, swelling until the equilibrium is reached. The swelling test was used to evaluate the absorption capabilities of non-crosslinked (CTH0) and crosslinked (CHT1) chitosan scaffolds in mixtures of a non-solvent (ethanol) and a solvent (water) - see Fig. 2. The combined effect of solvent and non-solvent in miscible liquid pairs is expected to be rather useful to control the swelling ratio within polymer networks. CHT, which is a semi-crystalline polymer, absorbs considerable amounts of water when immersed in aqueous environments. ${ }^{37}$ Ethanol was used as a non-solvent, aiming at providing adequate control over the CHT scaffolds' swelling capability.

With increasing water content, the swelling increases for both conditions. Below 30 vol\% of CHT1 and 50 vol\% of CHT0 such an increase is not evident, but above those values the swelling starts to increase prominently in both cases. These results agree with those of Ilavsky et al., who described polymeric hydrophilic networks in which a small variation in the composition of water-ethanol mixtures induces a jumpwise change in the volume of the gel, which is reflected by a simultaneous jumpwise change in the shear modulus. ${ }^{38}$ They also

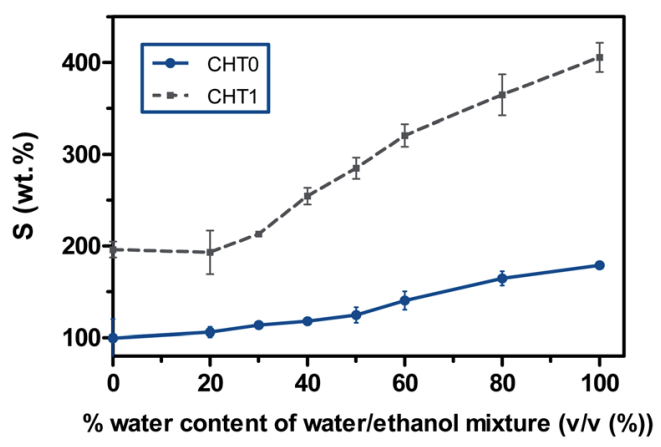

Fig. 2 Swelling capability of non-crosslinked (CHTO) and crosslinked (CHT1) chitosan scaffolds determined after immersion in distinct water-ethanol mixtures. found that in those types of systems the mechanical behaviour was predominantly determined by the degree of swelling: the jumpwise change in the modulus adequately correlated with the jump in the swelling ratio. In the case under study, a jump in swelling ratio of the CHT scaffolds is a strong indication that the referred type of volume transition occurs under the experimental conditions.

The swelling in the CHT scaffolds could be attributed to their intrinsic moderate hydrophilicity and also to the presence of pores that can be filled with liquid. The swelling results show that CHT1 presents higher solvent uptake capability than CHT0 in all water-ethanol compositions. The maximum swelling, reached for $100 \mathrm{vol} \%$ of water, is 179 vol\% for CHT0 and for CHT1 it is more than twice that for CHT0, 405\%. For the case of pure ethanol also the swelling in CHT1 is about two times that in CHT0. The observed differences could be a consequence of the different porous microstructure, especially the higher porosity of CHT1 as compared with CHT0, and also because genipin that was used to cross-link CHT1 exhibits high affinity to both water and ethanol.

The effect of swelling was also observed in X-ray diffraction analysis of CHT0. Fig. 3 shows the diffraction patterns of the CHT0 scaffolds immersed in different mixtures of waterethanol. A strong reflexion at $2 \theta=19$ is observed for dehydrated chitosan ( $0 \%$ water) in the diffraction patterns for CHT0, which evidences the semi-crystalline nature of CHT. With the increasing of water content, the relative intensity of reflection was significantly diminished. This is particularly evident above $50 \mathrm{vol} \%$ where an amorphous halo entered at about $2 \theta=28^{\circ}$ is highly visible. This occurrence can be explained by the jump of swelling for CHT0 observed at 50 vol\% - see Fig. 2. This increase of solvent content in the polymeric matrix hides the crystalline diffraction pattern of chitosan. Nevertheless, the semi-crystalline nature of CHT is maintained even upon immersion in pure water. The crystalline domains act as anchorage points for the amorphous fraction, being a necessary requirement for shape memory ability.

\subsection{Dynamic mechanical analysis measurements}

In biomedical applications, chitosan can be exposed to different levels of hydration, which can vary from moderate humidity levels to maximum values of water uptake capability, for example, under implantable conditions. The influence of water

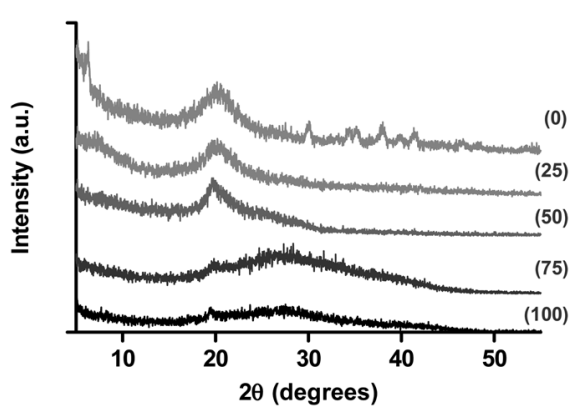

Fig. 3 XRD pattern for $\mathrm{CHTO}$ immersed in different mixtures of waterethanol from $0 \%$ to $100 \mathrm{v} / \mathrm{v} \%$ of water. 
content on the viscoelastic properties of chitosan films was already studied and was observed that chitosan can undergo a glass transition at room/body temperatures by the action of hydration. ${ }^{32,33}$

Dynamic mechanical analysis, DMA, was used to determine the storage modulus $\left(E^{\prime}\right)$ and loss factor $(\tan \delta)$ of CHT0 and CHT1 scaffolds as a function of water content. The chitosan scaffolds were placed in the DMA apparatus and immersed in an ethanol solution. The DMA parameters were then continuously monitored at $1 \mathrm{~Hz}$. During the measurements water was introduced into the reservoir at a constant flow rate while changing gradually the composition of the mixture (eqn (2)). Fig. 4 shows the variation of the storage modulus and the loss factor as a function of the water composition in the liquid mixture of the bath. The storage modulus of both samples shows a decrease with the increase of water content, with a profile suggesting the occurrence of a relaxation process induced by enriching the water content in the chitosan structure. This decrease of storage modulus with increasing water content was also observed in chitosan membranes immersed in mixtures of water-ethanol. ${ }^{33}$ The samples show a plateau storage modulus at water content of above 28.5 vol\% for CHT0 and 39.1 vol\% for CHT1. In the dehydrated state CHT1 exhibits the highest storage modulus of $c a .40 \mathrm{MPa}$ in comparison with ca. $17 \mathrm{MPa}$ of CHT0. With increasing water content the $\tan \delta$ curve of the samples exhibits a broad relaxation process that appears to be characterized by two components. Dielectric relaxation experiments performed at different temperatures and frequencies pointed out the complex relaxation pattern of chitosan. $^{39-41}$ However, the segmental mobility was never accessed before by this technique at different hydration levels.

The first component of the relaxation process that occurred in the range from 17 vol\% to 25 vol\% with a peak maximum around $21 \mathrm{vol} \%$ is attributed to the glass transition of the fraction of amorphous domains, which need lower water content to transit from the glassy to the rubbery state. The component of the relaxation process taking place at higher water content appears as a pronounced peak that reflects the glass transition of the amorphous domains confined in more restricted regions that are influenced by hard domains (crystalline and crosslinked environments). The occurrence of two glass transitions can be observed in semi-crystalline polymers reflecting co-operative segmental mobility of the amorphous chains in the non-confined bulk and from the macromolecular chains with restricted mobility due to geometrical confinements generated by the crystalline nanostructures. ${ }^{42}$ For CHT0 the peak is seen at ca. $32 \mathrm{vol} \%$ of water and for CHT1 it is at 40 vol\%. Above such water contents, i.e. above glass transition, the storage modulus is almost constant and the rubbery state of the polymeric structure is obtained. It is possible to see that CHT1 has a peak shifted towards higher water contents, indicating that the glass transition takes place for an increase of approximately $10 \mathrm{vol} \%$ of water. This could be explained by the effect of crosslinking that influences the dynamics (slowing down) of the segmental motions. ${ }^{43}$

\subsection{Shape memory behaviour of chitosan}

Several authors proposed the use of SMPs to fabricate fillers for pathological defect reparation to permit the implantation using less invasive procedures and to maximize the geometrical adaptation of the material in the defect cavity. ${ }^{\mathbf{9 , 4} 4}$ The interest in this type of material arises from the fact that SMPs can be deformed from a pre-defined shape into a stable temporary shape, significantly smaller, and can recover the original shape by some adequate stimulus. Therefore, evaluation of the shape recovery ability, in terms of final recovery, recovery rates and fixating ability, is hence mandatory.

The first test performed to study the shape memory effect of chitosan scaffolds was the water-driven recovery. The hydrated CHT0 was compressed under different maximum strains $\left(\varepsilon_{\mathrm{m}}\right)$ and retained this strain during dehydration to fix the temporary shape. After immersing the sample in water at room temperature, it started immediately to recover. The results, as shown in Fig. 5, demonstrate that the recovery occurs essentially in the first $5 \mathrm{~min}$ for the different $\varepsilon_{\mathrm{m}}$. After approximately 15 minutes, the samples have already reached the final shape. The increase of the maximum strain leads to a small decrease of the recovery ratio.

The influence of the applied $\varepsilon_{\mathrm{m}}$ on the shape memory performance of CHT scaffolds was investigated by hydromechanical tests with $\varepsilon_{\mathrm{m}}=10,20,30$, and $60 \%$. The shape fixity ratio $R_{\mathrm{f}}$ and the shape recovery ratio $R_{\mathrm{r}}$ were calculated to quantify the fixation of the temporary shape and the recovery of the permanent shape of the polymer networks. Both CHT0 and CHT1 scaffolds exhibited excellent shape memory properties as summarized in Table 1. A fixation of the deformation with $R_{\mathrm{f}} \geq$ 97.2\% was obtained for CHT0 and an almost complete fixation
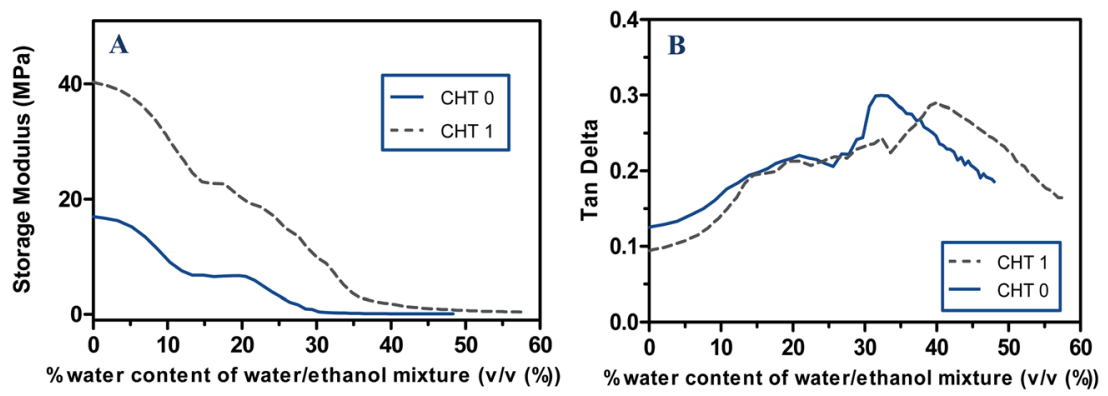

Fig. 4 Apparent storage modulus (A) and loss factor (B) measured with samples immersed in water-ethanol mixtures for CHTO and CHT1 at $1 \mathrm{~Hz}$. 


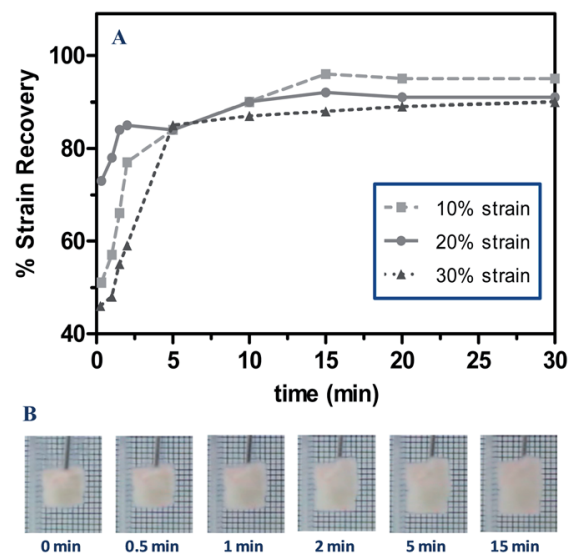

Fig. 5 (A) CHTO shape recovery along time after different maximum strains $\left(\varepsilon_{m}\right)$. (B) Series of photographs demonstrating the shape recovery process for $\mathrm{CHTO}$; the refereed times indicate the immersion time in water upon deformation at $\varepsilon_{\mathrm{m}}=30 \%$ and dehydration.

Table 1 Shape memory properties of CHTO and CHT1 at different deformation strains $\left(\varepsilon_{\mathrm{m}}\right)$

\begin{tabular}{llll}
\hline & & $\begin{array}{l}\text { Shape fixity ratio } \\
\left(R_{\mathrm{f}}\right)(\%)\end{array}$ & $\begin{array}{l}\text { Shape recovery } \\
\text { ratio }\left(R_{\mathrm{r}}\right)(\%)\end{array}$ \\
\hline \multirow{2}{*}{ CHT0 } & $\varepsilon_{\mathrm{m}}$ & $98.4 \pm 4.2$ & $96.8 \pm 3.6$ \\
& $10 \%$ & $97.7 \pm 2.9$ & $91.8 \pm 1.5$ \\
& $20 \%$ & $97.2 \pm 1.9$ & $87.5 \pm 2.8$ \\
& $30 \%$ & $98.8 \pm 0.2$ & $70.5 \pm 5.5$ \\
CHT1 & $60 \%$ & $100 \pm 0.6$ & $100 \pm 0.8$ \\
& $10 \%$ & $99.8 \pm 1.0$ & $99.1 \pm 2.3$ \\
& $20 \%$ & $99.2 \pm 0.5$ & $98.5 \pm 1.7$ \\
& $30 \%$ & $99.0 \pm 1.9$ & $98.7 \pm 1.2$ \\
\hline
\end{tabular}

with $R_{\mathrm{f}} \geq 99.2 \%$ was observed for CHT1, while the recovery of the original shape occurred with high $R_{\mathrm{r}}$ values: $70.5 \%$ for CHT0 and $98.5 \%$ for CHT1.

The shape recovery ratio increased with decreasing maximum strain. Too high deformation amplitude would make the shape memory effect less efficient for CHTO. Such an effect is not seen in CHT1. This result indicates that the crystalline structure in chitosan which affords all the shape recovery force in CHT0 may not be completely effective for large deformation strains. However, the crosslinking with genipin provides extra- anchorage points enhancing the recovery capability of the structure to the permanent shape.

Fig. 6A shows the variation of shape recovery along hydration in CHT0 for different deformation strains. The scaffolds do not exhibit significant recovery for water contents below 25 vol\%. The shape recovery starts between 25 and 50 vol\% having a drastic increase in the range of 50-75 vol\%, mainly for 20 and $30 \%$ strain. Such results are consistent with the swelling results in Fig. 2 and the occurrence of glass transition as seen by DMA (Fig. 4). As mentioned earlier, the shape recovery ratio decreased with increasing maximum strain and for $60 \%$ strain the recovery is unsatisfactory for CHTO that did not reach the permanent shape upon complete hydration. Therefore, in the following studies of the shape memory effect of CHT scaffolds, the maximum deformation strain was set as 30\%. However, crosslinked CHT presents better mechanical properties, as well as excellent recoverability - see Table 1 and Fig. 6B.

\subsection{Young's modulus of the scaffolds at distinct hydration levels}

The Young's modulus $(E)$ of the developed scaffolds upon immersion in water-ethanol mixtures was assessed via uniaxial compressive tests, obtained from the slope of the stress-strain curve. Fig. 7 shows an inverse relationship between $E$ and water content of the liquid. Water acts as a very good plasticizer even in small quantities. In the presence of water, the interference between water and the chain-to-chain secondary bonding reduces the intermolecular forces. As a result, chains acquire greater mobility and the free volume increases, leading to a decrease in the glass transition temperature and stiffness. ${ }^{45}$ Such a process is in the origin of the occurrence of the glass transition of the system as discussed before. Increasing the plasticizer content resulted in scaffolds with lower $E$ and high flexibility.

For both scaffolds, CHT0 and CHT1, as the water content increased, $E$ systematically decreased. The scaffolds exhibited water-dependent Young's modulus from 40.5 to $0.2 \mathrm{kPa}$. The values obtained for the dehydrated scaffolds are in accordance with the values obtained in previous reports for chitosan scaffolds in the dry state. ${ }^{46}$ With the decrease of water content, the increase of $E$ starts, in the vicinity of the glass transition event, for water contents below approximately $40 \%$. For water contents above $40 \%$ all the samples show a plateau in the modulus,
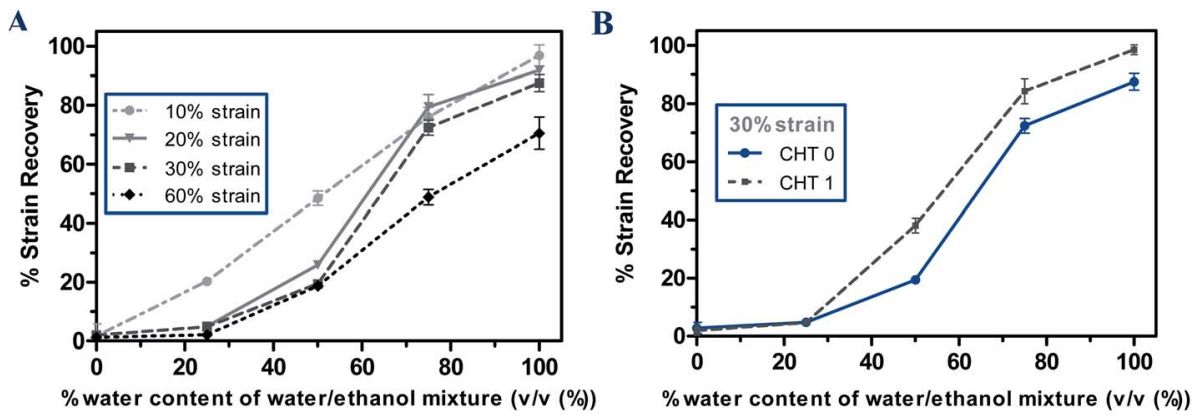

Fig. 6 (A) Strain recovery of $\mathrm{CHTO}$ in different mixtures of water-ethanol after different $\varepsilon_{\mathrm{m}}$. (B) Strain recovery of $\mathrm{CHTO}$ and $\mathrm{CHT} 1$ for $\varepsilon_{\mathrm{m}}=30 \%$. 


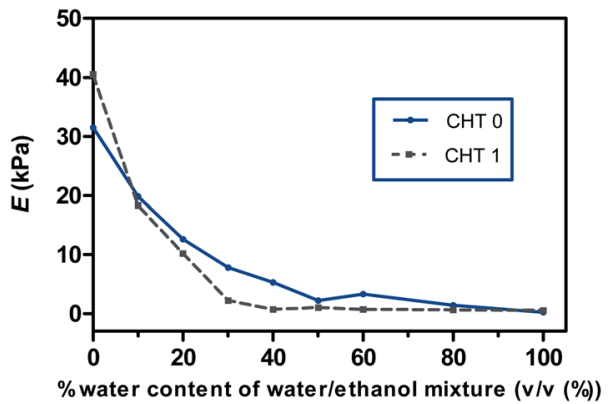

Fig. 7 The variation of Young's modulus with water-ethanol mixtures for $\mathrm{CHTO}$ and $\mathrm{CHT1}$.

suggesting a rubber like structure where the mobile chains in the amorphous regions are sustained by the crystalline and crosslinked domains.

\subsection{Hydromechanical compressive cycle}

To obtain more detailed shape memory properties of crosslinked and un-crosslinked CHT scaffolds, a new concept of hydromechanical cyclic compressive tests was introduced. The ensemble of the three-dimensional stress-strain-hydration response of the studied CHT0 and CHT1 scaffolds under uniaxial loading is shown in Fig. 8 III The cycle starts by hydrating the scaffolds to reach humidity above the glass transition, followed by a compression at a constant strain rate, resulting in a continuous stress-strain curve (curve I in Fig. 8). Then the temporary shape is fixed by dehydrating the scaffolds at constant compression $\left(\varepsilon_{\mathrm{m}}=30 \%\right)$ (stage II in Fig. 8). Then, the compressive stress is reduced until a stress-free condition is reached (stage III in Fig. 8). The scaffold is finally hydrated while the compressive stress is kept constant at $0 \mathrm{kPa}$ (sequence IV in

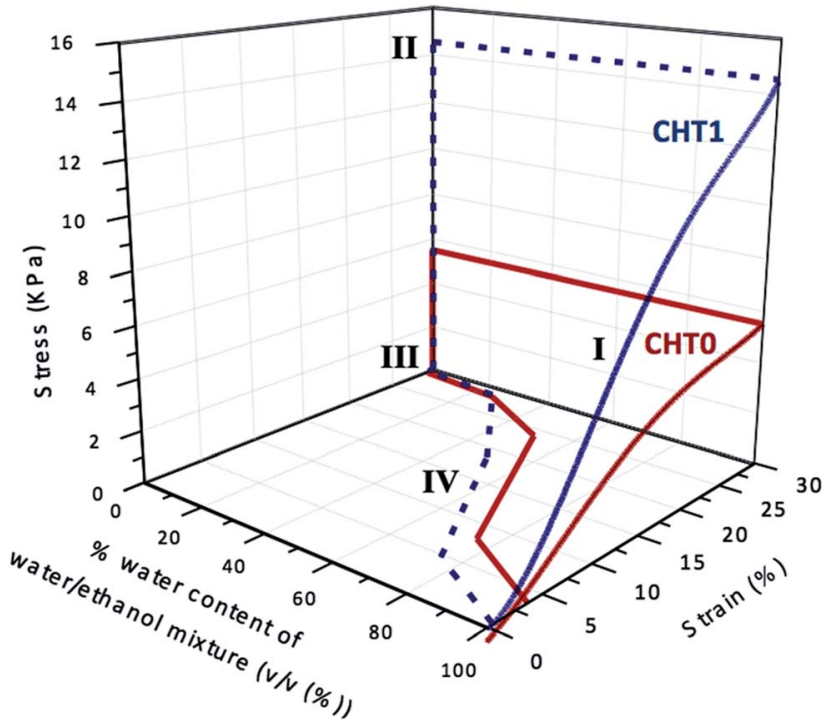

Fig. 8 Hydromechanical compressive cycle of CHT and CHT-BGNPs. (I) Compression at a constant rate of the hydrated sample; (II) dehydration of the sample at a fixed strain; (III) release of the stress; (IV) hydration in water-ethanol mixtures with increasing content of water.
Fig. 8). Such a process was performed by immersing the scaffolds in water-ethanol mixtures with increasing water content. The permanent shape is recovered while passing the glass transition, resulting in the strain-water content relationship.

The stress-strain curves were performed for CHT0 and CHT1 under hydrated conditions by compression tests up to $\varepsilon_{\mathrm{m}}=$ $30 \%$. The scaffolds exhibited the typical response observed in soft cellular materials for relatively low deformations. Compared to CHT0 scaffolds, CHT1 achieves a higher compressive stress at $\varepsilon_{\mathrm{m}}=30 \%$ and the slope of the initial straight line is also higher than CTH0, confirming that CHT1 has a higher compressive modulus $(E)$. After loading, $\varepsilon_{\mathrm{m}}$ is maintained while the samples are dehydrated. When the stress is removed the scaffolds reach the temporary shape.

The strain-hydration curves present in the compressive cycle were obtained by hydration of the dehydrated scaffolds in mixtures of water and ethanol, varying from pure ethanol to pure water. In the hydration process, the strain is recovered significantly above $40 \%$ of water content, for a water content rate above the glass transition occurrence.

\subsection{In vitro drug delivery studies}

The potential of the system for loading and in situ release of drugs was explored in order to achieve a multifunctional polymer system with the SME, biodegradability and controlled drug release. In this study, Congo Red (CR) was selected as a model molecule and was loaded in the scaffolds by swelling. CR is water soluble and presents low solubility in ethanol. At physiological $\mathrm{pH} \mathrm{CR}$ acquires an anionic form, as the sulfonate groups gets negatively charged (see the structure of this molecule in Fig. 9C), and thus the molecule could be considered as a model for many small anionic drugs. Given the typical cationic nature of chitosan, we expect some favourable interaction between the chains of this biopolymer and CR molecules.

The swelling of the scaffolds in CR solutions at room temperature permits the introduction of CR into the polymer matrix. With this technique the loading is dependent on both the CR concentration and the swelling of the polymer network in the solution. After loading, the scaffolds were compressed and dehydrated under stress to reach a temporary shape. Then, the release studies with CHTO and CHT1 loaded with CR were conducted at $37^{\circ} \mathrm{C}$ in PBS. Previously, we analysed the influence on the loading capability of CR in the two solvents, water and PBS. We verified that no differences could be observed. We also observed no differences in swelling when CR was added in PBS, as compared with pure water.

Fig. 9A presents the release profiles for CHT0 and CHT1 for $48 \mathrm{~h}$. For both scaffolds, CR was released relatively quickly up to 2 hours and then the release rate slowed with time. The initial fast release observed in both profiles can be attributed to the presence of CR on (or close to) the scaffold surface or the surface of the pore walls. The ability of the scaffolds to swell leads to the increase of the pore size in the scaffold structure that allows the diffusion of CR into the PBS solution. Thus, as CHT1 presents better water uptake capability it also releases more CR than CHT0. 

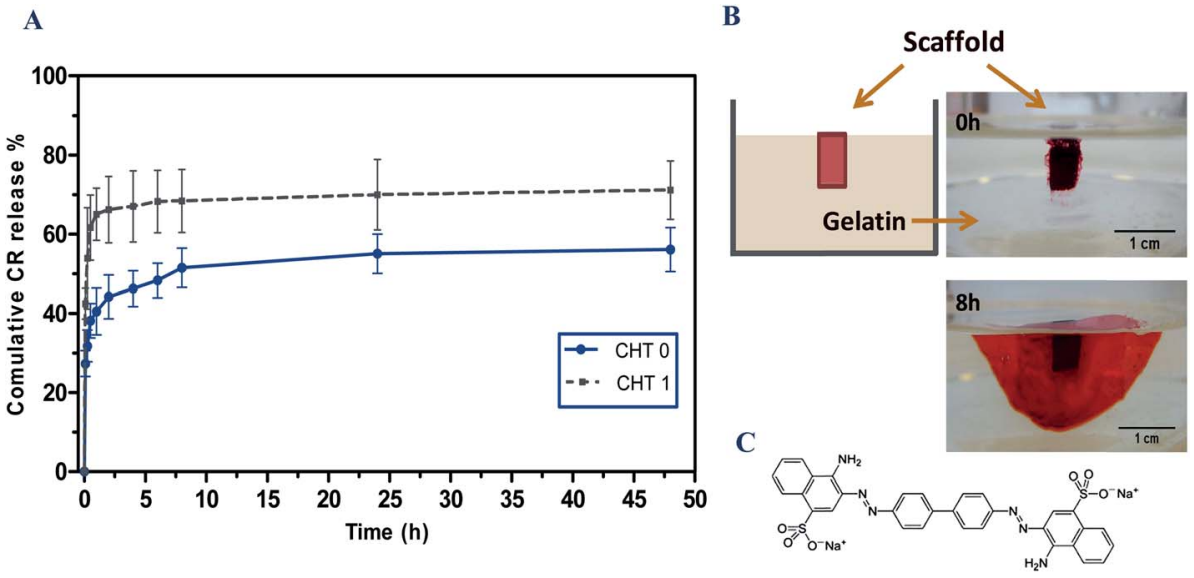

Fig. 9 (A) In vitro release of $\mathrm{CR}$ as a function of time for $\mathrm{CHTO}$ and $\mathrm{CHT} 1$ in $\mathrm{PBS}\left(37^{\circ} \mathrm{C}\right)$. (B) Images showing $\mathrm{CR}$ being released from a $\mathrm{CHTO}$ scaffold after being placed in a defect produced in a gelatin block at two distinct time points. (C) Congo red structure.

We envisage the possibility of using such technology to deliver therapeutic drugs to specific sites in the body. As an example, we used gelatin as a continuous medium simulating a soft tissue. A cylindrical defect in a gelatin block was produced with a size between the permanent and temporary sizes of the scaffolds. The scaffolds containing CR (temporary shape) were placed in the region of the empty space. Due to the hydrated environment the scaffold swells inside the defect, adapting the local geometry and starting to release CR. Fig. 9B shows an image of the system after the introduction of the scaffold and after $8 \mathrm{~h}$ of release. After this period of time it is possible to see a gradient with higher concentration of CR next to the scaffolds, indicating the diffusion of the molecule into the medium. This result reveals that the developed shape memory device can be potentially used as a drug loading matrix. Moreover it could also be observed that upon recovery in the hydrated state the scaffold was able to accommodate perfectly in the entire geometry of the defect site and could be maintained tightly in this volume due to a press fitting effect.

\section{Conclusions}

Semi-crystalline (CHT0) and chemically crosslinked (CHT1) CHT scaffolds showed a shape memory effect using hydration as the stimulus. CTH0 exhibits a glass transition for $32.3 \mathrm{vol} \%$ of water in water-ethanol mixtures and CHT1 shows this effect at $40 \mathrm{vol} \%$. Both scaffolds possess good shape memory properties: a fixity ratio above $97.2 \%$ for CHT0 and above $99.2 \%$ for CHT1 and a recovery ratio above $70.5 \%$ for CHT0 and $98.5 \%$ for CHT1. The shape memory properties decreased with increasing maximum deformation. However, CHT1 presents better mechanical properties and excellent recoverability even for high deformations. The release profiles of CR from the scaffolds show that the developed porous structures could be used as a controlled delivery system. These scaffolds could be interesting in applications where 3D devices should be implanted in empty and hydrated regions. Examples could be bone or cartilage defects. The scaffold could be introduced, for example, inside low diameter tubes, where they could be confined in a temporary shape with a smaller size than the permanent one. At the implantation site the scaffolds could be pushed out from the tubes and the natural hydrated environment could drive the recovery of the desired shape. This recovery of the shape could also produce some press-fitting pressure that could help in the fixation of the implant. We can conclude that the CHT scaffolds proposed in this work are candidates for applications in minimally invasive surgery with multifunctional characteristics, combining biodegradability, shape-memory capability and controlled drug release.

\section{Acknowledgements}

This work was supported by the Portuguese Foundation for Science and Technology Foundation (FCT) through project PTDC/FIS/115048/2009. We acknowledge Dr Ana Rita Duarte for all the help during this project and Joana Marques Silva and Sofia Caridade for their contribution to the DMA experiments.

\section{References}

1 C. Wischke, A. T. Neffe and A. Lendlein, Adv. Polym. Sci., 2010, 226, 177-205.

2 M. Behl, M. Y. Razzaq and A. Lendlein, Adv. Mater., 2010, 22, 3388-3410.

3 M. C. Serrano and G. A. Ameer, Macromol. Biosci., 2012, 12, 1156-1171.

4 A. Lendlein, Shape-Mem. Polym. Multifunct. Compos., 2009, 226.

5 F. Liu and M. W. Urban, Prog. Polym. Sci., 2010, 35, 3-23.

6 I. A. Rousseau, Polym. Eng. Sci., 2008, 48, 2075-2089.

7 N. Choi and A. Lendlein, Soft Matter, 2007, 3, 901-909.

8 B. Yang, W. Min Huang, C. Li and J. Hoe Chor, Eur. Polym. J., 2005, 41, 1123-1128.

9 A. Lendlein and S. Kelch, Angew. Chem., Int. Ed., 2002, 41, 2034-2057.

10 E. Ghobadi, M. Heuchel, K. Kratz and A. Lendlein, Polymer, 2013, 54, 4204-4211. 
11 A. Lendlein, H. Jiang, O. Jünger and R. Langer, Nature, 2005, 434, 879-882.

12 T. Weigel, R. Mohr and A. Lendlein, Smart Mater. Struct., 2009, 18, 025011.

13 J. W. Cho, J. W. Kim, Y. C. Jung and N. S. Goo, Macromol. Rapid Commun., 2005, 26, 412-416.

14 H. Zhang, H. Wang, W. Zhong and Q. Du, Polymer, 2009, 50, 1596-1601.

15 S. Chen, J. Hu and S. Chen, Polym. Int., 2012, 61, 314-320.

16 G. Niu and D. Cohn, Science, 2013, 3, 49-50.

17 W. Huang, B. Yang, Y. Zhao and Z. Ding, J. Mater. Chem., 2010, 20, 3367-3381.

18 H. Du and J. Zhang, Soft Matter, 2010, 6, 3370-3376.

19 K. Fan, W. M. Huang, C. C. Wang, Z. Ding, Y. Zhao, H. Purnawali, K. C. Liew and L. X. Zheng, eXPRESS Polym. Lett., 2011, 5, 409-416.

20 C. C. Wang, W. M. Huang, Z. Ding, Y. Zhao and H. Purnawali, Compos. Sci. Technol., 2012, 72, 11781182.

21 B. Yang, W. Huang, C. Li, C. Lee and L. Li, Smart Mater. Struct., 2004, 13, 191.

22 B. F. Pierce, K. Bellin, M. Behl and A. Lendlein, Int. J. Artif. Organs, 2011, 34, 172-179.

23 H. Lv, J. Leng, Y. Liu and S. Du, Adv. Eng. Mater., 2008, 10, 592-595.

24 L. De Nardo, S. Farè, S. D. Cicco, M. Jovenitti and M. C. Tanzi, Mater. Sci. Forum, 2007, 663-668.

25 Y. Wong, Y. Xiong, S. Venkatraman and F. Boey, J. Biomater. Sci., Polym. Ed., 2008, 19, 175-191.

26 M. Bao, Q. Zhou, W. Dong, X. Lou and Y. Zhang, Biomacromolecules, 2013, 14, 1971-1979.

27 N. Alves and J. F. Mano, Int. J. Biol. Macromol., 2008, 43, 401414.

28 P. J. VandeVord, H. W. T. Matthew, S. P. DeSilva, L. Mayton, B. Wu and P. H. Wooley, J. Biomed. Mater. Res., 2002, 59, 585590.
29 I.-Y. Kim, S.-J. Seo, H.-S. Moon, M.-K. Yoo, I.-Y. Park, B.-C. Kim and C.-S. Cho, Biotechnol. Adv., 2008, 26, 1-21.

30 A. Di Martino, M. Sittinger and M. V. Risbud, Biomaterials, 2005, 26, 5983-5990.

31 J. F. Mano, G. A. Silva, H. S. Azevedo, P. B. Malafaya, R. A. Sousa, S. S. Silva, L. F. Boesel, J. M. Oliveira, T. C. Santos, A. P. Marques, N. M. Neves and R. L. Reis, J. R. Soc. Interface, 2007, 4, 999-1030.

32 J. F. Mano, Macromol. Biosci., 2008, 8, 69-76.

33 S. G. Caridade, R. M. da Silva, R. L. Reis and J. F. Mano, Carbohydr. Polym., 2009, 75, 651-659.

34 J. Jin, M. Song and D. Hourston, Biomacromolecules, 2004, 5, 162-168.

35 R. M. Teather and P. J. Wood, Appl. Environ. Microbiol., 1982, 43, 777-780.

36 L. Ma, C. Gao, Z. Mao, J. Zhou, J. Shen, X. Hu and C. Han, Biomaterials, 2003, 24, 4833-4841.

37 R. Silva, G. Silva, O. Coutinho, J. F. Mano and R. R. Reis, J. Mater. Sci.: Mater. Med., 2004, 15, 1105-1112.

38 M. Ilavsky, Macromolecules, 1982, 15, 782-788.

39 M. Viciosa, M. Dionisio, R. Silva, R. R. Reis and J. F. Mano, Biomacromolecules, 2004, 5, 2073-2078.

40 M. Viciosa, M. Dionísio and J. F. Mano, Biopolymers, 2006, 81, 149-159.

41 C. Gartner, B. L. López, L. Sierra, R. Graf, H. W. Spiess and M. Gaborieau, Biomacromolecules, 2011, 12, 1380-1386.

42 Y. Wang, J. Gómez Ribelles, M. Salmerón Sánchez and J. F. Mano, Macromolecules, 2005, 38, 4712-4718.

43 N. Alves, G. Ribelles, G. Tejedor and J. F. Mano, Macromolecules, 2004, 37, 3735-3744.

44 A. Lendlein, M. Behl, B. Hiebl and C. Wischke, Expert Rev. Med. Devices, 2010, 7, 357-379.

45 B. Yang, W. Huang, C. Li and L. Li, Polymer, 2006, 47, 13481356.

46 M. Liu, C. Wu, Y. Jiao, S. Xiong and C. Zhou, J. Mater. Chem. $B, 2013,1,2078-2089$. 